\title{
Time-Variant Reliability Analysis of Reinforced Concrete Structure Based on Method of Moments
}

\author{
Tao Fu ${ }^{1, \text { a }}$, Liqun Yuan ${ }^{2, \mathrm{~b}}$ and Chaobiao Gao ${ }^{1, \mathrm{c}}$ \\ ${ }^{1}$ School of Transportation Engineering, Shandong Jianzhu University; Jinan 250101, Shandong, \\ China; \\ ${ }^{2}$ School of Architecture and Civil Engineering, Liaocheng University; Liaocheng 252059, Shandong, \\ China; \\ agreenvillage_17@163.com, ${ }^{\mathrm{b}}$ liqunyuan@163.com, ' 9 gcbliterature@139.com
}

Keywords: time-variant reliability; method of moments; random process

Abstract: For the influence of corrosive environment, the reliability index of reinforced concrete structure will decrease in design reference period. A method of time-variant reliability is proposed based on method of moments. The resistance and load effect are described as random process. The random process is transformed into random variable by the method of equivalent resistance method. The time-variant reliability is carried on in design reference period accurately with consideration of characteristic of random variables which can provide supports for the management departments to take appropriate measures to reduce economic loss in the engineering circles.

\section{Introduction}

For the influence of corrosive environment, such as concrete carbonation and reinforcement corrosion, resistance of reinforced concrete structure will attenuate which brings difficulty to maintenance and management of structure. It also gives rise to enormous casualties, property loss and environmental destruction which forces researchers and officials to take a closer look at the structural damage and find methods to improve durability of concrete. So it has become a more and more concerned problem to carry on time-variant reliability analysis of reinforced concrete structure rationally to provide supports for the management departments to take appropriate measures to reduce economic loss in the engineering circles $^{[1]}$.

\section{Mathematical model of time-variant reliability analysis of reinforced concrete structure}

The reliability of structure is determined by two statistically independent random variables, load effect variable $S$ and resistance variable $R$. Load effect variable and resistance variable should be described as random process for its time varying characteristics. Therefore the performance function can be defined as follows:

$$
Z(t)=g[R(t), S(t)]=R(t)-S(t)
$$

The $R(t)$ denotes the random process of resistance, $S(t)$ denotes the random process of load effect.

The probability of safe in design reference period can be described as follows:

$$
P_{s}(T)=P\{Z(t)>0, t \subset[0, T]\}=P\{R(t)>S(t), t \subset[0, T]\}
$$

Where $T$ denotes design reference period.

The probability of failure in design reference period can be described as follows:

$$
P_{f}(T)=1-P_{s}(T)=P\{R(t) \leq S(t), t \subset[0, T]\}
$$

The failure of structure will occur if the resistance of structure is less than load effect at any time in design reference period.

Alternatively, the performance function can be described as follows:

$$
Z(t)=R(t)-S_{G}-S_{Q}(t)
$$

Where $S_{G}$ denotes permanent load effect, $S_{Q}(t)$ denotes the random process of variable load effect. 
Theoretically, the failure probability is described as:

$$
P_{f}(T)=P\left\{R\left(t_{i}\right)-S_{G}-S_{Q}\left(t_{i}\right)<0, t_{i} \subset[0, T]\right\}=P\left\{\min \left[R(t)-S_{G}-S_{Q}(t)\right]<0, t \subset[0, T]\right\}
$$

Considering the attenuation of resistance of reinforced concrete structure, the random process of resistance is give as ${ }^{[2]}$ :

$$
R(t)=R_{0} \varphi(t)
$$

Where $R_{0}$ denotes the resistance of structure for $t=0$.

$\varphi(t)$ is a deterministic function.

Corresponding, the mean and coefficient of variation can be expressed as:

$$
\begin{aligned}
& \mu_{R(t)}=\mu_{R_{0}} \varphi(t) \\
& \sigma_{R(t)}=\sigma_{R_{0}}
\end{aligned}
$$

Time-variant reliability analysis can be conducted on condition that the random process should be transformed into random variable. In this paper, the random process is transformed into random variable by the method of equivalent resistance method.

First of all, design reference period is divided into m periods which can be described as $\tau=T / \mathrm{m}$.

Then the random process of load effect and resistance are dispersed into $\mathrm{m}$ random variables. The failure probability is expressed as:

$$
\begin{aligned}
& P_{f}(T)=P\left\{\min \left[R\left(t_{i}\right)-S_{G}-S_{Q}\left(t_{i}\right)\right]<0, t_{i}=(i-0.5) \tau, i=1,2, \cdots m\right\} \\
& =P\left\{\bigcup_{i=1}^{m} R\left(t_{i}\right)-S_{G}-S_{Q}\left(t_{i}\right)<0, t_{i}=(i-0.5) \tau, i=1,2, \cdots m\right\} \\
& =P\left\{\bigcup_{i=1}^{m} R_{i}-S_{G}-S_{Q i}\left(t_{i}\right)<0\right\}
\end{aligned}
$$

where $S_{Q i}$ denotes the ith random variable of load effect, $R_{i}$ denotes the ith random variable of resistance. The value of $R_{i}$ is taken at the middle of every period ${ }^{[3]}$.

This is a structural reliability analysis of series system. If the maximum value of load effect is a Gumbel random variable, the performance function for time-variant reliability analysis can be formulated as follows ${ }^{[4]}$ :

$$
\begin{aligned}
& P_{f}(T)=P\left\{\min \left[R\left(t_{i}\right)-S_{G}-S_{Q}\left(t_{i}\right)\right]<0, t_{i}=(i-0.5) \tau, i=1,2, \cdots m\right\} \\
& =P\left\{\bigcup_{i=1}^{m} R\left(t_{i}\right)-S_{G}-S_{Q}\left(t_{i}\right)<0, t_{i}=(i-0.5) \tau, i=1,2, \cdots m\right\} \\
& =P\left\{\bigcup_{i=1}^{m} R_{i}-S_{G}-S_{Q i}\left(t_{i}\right)<0\right\}
\end{aligned}
$$

where $R=-\frac{1}{\alpha_{T}} \ln \left[\frac{1}{m} \sum_{i=1}^{m} \exp \left(-\alpha_{T} R_{i}\right)\right]$

$R$ denotes equivalent resistance which denotes the change law of resistance in design reference period.

\section{Method of moments for structural reliability}

The method of moments, being very simply, has no shortcoming with respect to design points, and requires neither iteration nor computation of derivatives and computation of mutual correlations among failure modes, and thus are convenient to be applied to structural reliability analysis ${ }^{[1]}$.

For a performance function $Z=G(X)$, using inverse Rosenblatt transformation, the kth moments about zero, of $\mathrm{Z}$ can be defined as ${ }^{[1,5]}$ : 


$$
\begin{aligned}
\mu_{k G} & =E\left\{[G(\mathbf{X})]^{k}\right\}=\int_{-\infty}^{\infty} \ldots \int_{-\infty}^{\infty}\{G[\mathbf{x}]\}^{k} f_{\mathbf{X}}(\mathbf{x}) d \mathbf{x} \\
& =\int_{-\infty}^{\infty} \ldots \int_{-\infty}^{\infty}\left\{G\left[T^{-1}(\mathbf{u})\right]\right\}^{k} \phi(\mathbf{u}) d \mathbf{u}
\end{aligned}
$$

Where $f_{x}(x)$ denotes the probability density function (PDF) of $\mathbf{X} . T^{-1}(u)$ denotes inverse Rosenblatt transformation. $\phi(u)$ denotes the PDF of standard normal variables.

According the dimension reduction integration(DRI) for moments of performance function ${ }^{[6]}$, the mean, standard deviation, the skewness, and the kurtosis of a performance function $G(X)$ with $n$ random variables can be obtained as the follows:

$$
\begin{aligned}
\mu_{G} & =\mu_{1} \\
\sigma_{G} & =\sqrt{\mu_{2}-\mu_{1}^{2}} \\
\alpha_{3 G} & =\left(\mu_{3}-3 \mu_{2} \mu_{1}+2 \mu_{1}^{2}\right) / \sigma_{G}{ }^{3} \\
\alpha_{4 G} & =\left(\mu_{4}-4 \mu_{3} \mu_{1}-3 \mu_{2}{ }^{2}+12 \mu_{2} \mu_{1}{ }^{2}-6 \mu_{2}{ }^{4}\right) / \sigma_{G}{ }^{4}
\end{aligned}
$$

The $\mu_{i}$ denotes the $k$ th moments about zero which can be calculated by point estimate method with estimating points and the corresponding weights ${ }^{[1,7]}$

With the first few moments of the performance function obtained by DRI, the moment-based reliability index can be evaluated as follows ${ }^{[1,7]}$ :

$$
\begin{aligned}
& \beta_{2 M}=\mu_{G} / \sigma_{G} \\
& \beta_{3 M}=\left(3-\sqrt{9+\alpha_{3 G}-6 \alpha_{3 G} \beta_{2 M}}\right) / \alpha_{3 G} \\
& \beta_{4 M}=-\Phi^{-1}\left[\int_{-\infty}^{\beta_{2 M}} f\left(Z_{u}\right) d Z_{u}\right], Z_{u}=\left(Z-\mu_{G}\right) / \sigma_{G}
\end{aligned}
$$

Then the failure probability can be obtained.

\section{Application in practical engineering}

A detailed example of time-variant reliability analysis is presented in this paper.

The random process of normal section bearing capacity of beam is expressed as $R(t)=R_{0} \varphi(t)$. where $\varphi(t)=1-2.2 \times 10^{-6} t^{3}$. The design reference period is taken as 50 years.

The taken values of parameters for time-variant reliability analysis are listed in Table 1.

Table. 1 The value of analysis parameters

\begin{tabular}{cccc}
\hline Parameter & Distribution type & Mean & Variation coefficient \\
\hline$R_{0}$ & lognormal distribution & $381 \mathrm{kN} \cdot \mathrm{m}$ & 0.1 \\
$S_{G}$ & normal distribution & $66.25 \mathrm{kN} \cdot \mathrm{m}$ & 0.072 \\
$S_{Q T}$ & extreme type I distribution & $99.44 \mathrm{kN} \cdot \mathrm{m}$ & 0.287 \\
\hline
\end{tabular}

As for calculation the time-variant reliability of the beam, the design reference period is divided into 5 periods which can be calculated as $\tau=50 / 5=10$.

The random process of resistance is dispersed into 5 random variables.

$$
R_{i}=R_{0} \varphi\left(t_{i}\right) \quad t_{i}=(i-0.5) \tau, i=1,2, \cdots 5
$$

The performance function of structure can be formulated as follows:

$$
g\left(R_{0}, S_{G}, S_{Q T}\right)=-\frac{1}{\alpha_{T}} \ln \left[\frac{1}{m} \sum_{i=1}^{m} \exp \left(-\alpha_{T} R_{0} \varphi\left(t_{i}\right)\right)\right]-S_{G}-S_{Q T}
$$

The mean and standard deviation of extreme type I distribution can be calculated as follows:

$$
\mu_{S_{Q t}}=\mu_{S_{Q T}}+\frac{\ln \left(\frac{t}{T}\right)}{\alpha_{T}} \quad \sigma_{S_{Q t}}=\sigma_{S_{Q T}}
$$


The parameters of extreme type I distribution in design reference period are shown in Table 2 .

Table.2The parameters of variable load effect $(k N \llbracket m)$

\begin{tabular}{cccccc}
\hline time & $10^{\text {th }}$ year & $20^{\text {th }}$ year & $30^{\text {th }}$ year & $40^{\text {th }}$ year & $50^{\text {th }}$ year \\
\hline$\mu_{S_{Q t}}$ & 62.617 & 78.044 & 87.069 & 93.472 & 98.438 \\
$\sigma_{S_{Q t}}$ & 28.547 & 28.547 & 28.547 & 28.547 & 28.547 \\
\hline
\end{tabular}

The reliability index and probability of failure in design reference period are shown in Table 3 . Table.5 Reliability index and failure probability

\begin{tabular}{cccccc}
\hline time & $10^{\text {th }}$ year & $20^{\text {th }}$ year & $30^{\text {th }}$ year & $40^{\text {th }}$ year & $50^{\text {th }}$ year \\
\hline$\beta$ & 4.151 & 3.968 & 3.816 & 3.612 & 3.238 \\
$P_{f}(t)$ & $1.65 \mathrm{E}-05$ & $3.62 \mathrm{E}-05$ & $6.77 \mathrm{E}-05$ & $1.52 \mathrm{E}-04$ & $6.02 \mathrm{E}-04$ \\
\hline
\end{tabular}

\section{Conclusions}

The reliability index of structure will decrease in design reference period. A method of time-variant reliability is proposed based on method of moments. This method can reflect the change law of probability failure in design reference period accurately with consideration of characteristic of random variables. This method is suitable to be used in structural time-variant reliability analysis.

\section{Acknowledgements}

This work was financially supported by the Project of Shandong Province Higher Educational Science and Technology Program (J13LN62) and Project of Shandong Jianzhu University Doctoral Fund.

\section{References}

[1]Junjie Wang,Tao Fu,Yuanfeng Bao.Vessel-Bridge Collision Risk Analysis Based on Structural Reliability Theory.Journal of Asian Architecture and Building Engineering,8 (2) ,539-545,2009

[2]Y.Mori, B. R.Ellingwood. Maintaining realibility of concrete structure. I: Role of inspection/repair. Journal of Structure Engineering, 1993,120(3)

[3]D.G.Lu,X.P.Fan,W.Jiang.Comparisons of four time-dependent reliability approaches for safety assessment of deteriorate concrete bridge.Proceedings of the Sixth International IABMAS Conference, Stresa, Lake Maggiore, Italy, 8-12 July 2012

[4] Gong Jinxin. Reliability Based Durability Analysis of Reinforced Concrete Structures. Ph.D. thesis of Dalian University of Technology. 1999

[5] Zhao, Y.G. and Ono, T. New point-estimates for probability moments. Journal of Engrg, Mech., ASCE, 126(4), 433-436. 2000

[6] Zhao, Y.G. and Lin, Y.S. (2008) Dimension Reduction Integration for System Reliability. The 4th Asian-Pacific Symposium on Structural Reliability and its Application, 214-220.2008

[7] Zhao, Y.G. and Ono, T. (2001) Moment methods for structural reliability. Structural Safety, 23(1), 47-75.2001 